\title{
Marco jurídico legal de la desertificación: caso Tingopaccha, Junín
}

\author{
Judicial framework legal of desertification: \\ Tingopaccha, Junín case
}

\begin{abstract}
Isabel M. Castro Poma 1*, Camila F. Miranda Peralta ', Mariela M. Pedrosa Siméon ', Dante E. Rivera Romero ', Mary A. Sánchez Valentín ${ }^{1}$ y Emma C. Jaramillo Cabrera ${ }^{1}$

'Facultad de Derecho, Universidad Continental, Huancayo, Perú
\end{abstract}

\section{RESUMEN}

La presente investigación abarcó un estudio del marco jurídico, nacional e internacional, que regula un problema que se creía ajeno a nuestra realidad, la desertificación, consistente en la degradación de la tierra. Empero, como el Derecho tiene que ser analizado de forma casuística, setuvo como referencia el caso suscitado en la Comunidad campesina de Tingopaccha, anexo de Acolla, provincia de JaujaJunín, originado por el monocultivo de maca en grandes extensiones. Es así que, cuando se realizó el trabajo de campo se observó que el daño ocasionado en el paraje Juchupampa era más grave de lo mencionado en el expediente administrativo. No obstante, a pesar de lo mediático que se hizo este problema se pudo comprobar que subsiste, ya que se continúa realizando monocultivos de maca, incluso en tierras vírgenes. De ese modo, se pudo corroborar que existen muchos instrumentos legales que pueden hacerle frente a la desertificación, como: la Convención de las Naciones Unidas para la Lucha contra la Desertificación, el Plan Estratégico Decenal 2008-2018, la Ley Forestal y de Fauna Silvestre, la Estrategia Nacional de Lucha contra la Desertificación 2016-2030, entre otros. En conclusión el problema no reside en el ordenamiento jurídico sino en los operadores jurídicos que la aplican; asimismo, que para combatir esto, más allá de que exista una sanción pecuniaria, es necesario que se sancione esta conducta como delito contemplando como agravante el monocultivo.

Palabras clave: Degradación, monocultivo, maca, cambio de uso de la tierra, ecosistema.

\begin{abstract}
The present investigation included a study of the legal framework, national and international, that regulates a problem that was thought alien to our reality, desertification, consisting of the degradation of the earth. However, as the law has to be analyzed in a casuistic form, reference was made to the case raised in the peasant community of Tingopaccha, Acolla annex, province of Jauja-Junín, originated by the monoculture of maca in large extensions. So that, when the field work took place it was observed that damage in the places of Juchupampa, it was more serious than mentioned in the administrative file. Nevertheless, in spite of the mediatic nature of this problem, it could be proven that it still exists, since monocultures of maca are still being carried out, even in virgin lands. Nevertheless, in spite of the mediatic nature of this problem, it could be proven that it still exists, since monocultures of maca are still being carried out, even in virgin lands. In this way, it was possible to corroborate that there are many legal instruments that can cope with desertification, such as: the United Nations Convention to Combat Desertification, the 2008-2018 Strategic Plan, the Forestry and Wildlife Law, the National Strategy to Combat Desertifications 2016-2030, among others. In conclusion, the problem lies not in the legal system but in the legal operators that apply it; likewise, that in order to combat this, beyond the existence of a pecuniary sanction, it is necessary that this behavior be sanctioned as an offense, considering the monoculture as an aggravating circumstance.
\end{abstract}

Keywords: Degradation, monoculture, maca, change of land use, ecosystem.

Historial del artículo:

Recibido, 05 de junio de 2017; aceptado, 15 de junio de 2017; disponible en línea, 30 de junio de 2017

* Estudiante de la Universidad Continental.

Correo: 47203345@continental.edu.pe 


\section{INTRODUCCIÓN}

Tingopaccha, es una comunidad campesina que en el año 2014 arrendó a la empresa SILMAX E.I.R.L. más de 100 hectáreas de tierra para monocultivar maca en grandes extensiones, lo que trajo daños irreparables puesto que, la maca es una planta herbácea catalogada como un superalimento, la que al ser cultivada de esa manera trae consigo la desertificación de las tierras volviéndolas infértiles, derivando no sólo en un grave daño ambiental sino que también afecta a los pobladores que viven de la agricultura y la ganadería, situación que durará cerca de diez años, y si se continúa con dicha práctica la tierra terminará erosionándose, tal como acaeció en Guinea Ecuatorial. Es por ello, que la investigación realizada se orientó analizar el referido problema a la luz de la legislación nacional e internacional aplicable buscando identificar los factores por los que no se puede combatir con eficiencia este problema medio -ambiental, que cada vez más y más afecta a nuestro país.

La investigación se realizó en la comunidad campesina de Tingopaccha, situada en el distrito de Acolla a 23 $\mathrm{km}$ de la provincia de Jauja, departamento de Junín; cuya superficie total es de 10,9 km2, con una altitud media de 3,730 m.s.n.m., (Municipalidad distrital de Acolla, s.f.) dentro de las coordenadas geográficas $11^{\circ}$ $51^{\prime} 06^{\prime \prime}$ latitud sur y $75^{\circ} 30^{\prime} 27$ latitud oeste (Google Maps, 2018). Es así que, se situó el problema en el paraje de Juchapampa, en una extensión de 36,45 has. de tierras comunales.

\section{Ecología}

Según el SENAMHI (Servicio Nacional de Meteorología e Hidrología del Perú, 2011) el clima del lugar es templado y seco, con frío y lluvias en épocas invernales. La temperatura varía entre los $18,7^{\circ} \mathrm{C}$ y $7,5^{\circ} \mathrm{C}$ debido a su altitud y ubicación en la sierra central.

\section{Biodiversidad}

Flora. Tingopaccha ostenta una gran variedad de vegetales que son parte de la flora nativa y agrícola, entre las que resaltan las especies stipa ichu y festuca weberbaveri, consideradas Patrimonio Forestal Nacional conforme al artículo $33^{\circ}$ del Reglamento de la Ley Forestal y de Fauna Silvestre.

Que hacen de esta área geográfica una zona apta, generando el hábitat adecuado para la realización y mantenimiento ecológico vital para albergar la fauna que ostenta.

Fauna. La especie distintiva de la zona es la vicuña la cual se encuentra dentro de la reserva denominada
Reserva Natural de Lomo Largo - Tingopaccha como medio de protección a los animales y la sustentación de la biodiversidad de la zona.

Hidrografía. Tingopaccha presenta una precipitación pluvial aproximada de $650 \mathrm{~mm}$ anual, gracias por lo que cuenta con lagunillas, manantiales, diversos ojos de agua, tres riachuelos, y dos presas. Las cuales se encuentran como sustento de los campos con plantaciones de cebada, papa, etc. Y como fuente de abastecimiento de los animales silvestres protegidos en la reserva, así como también, de los animales denominados ganado.

\section{MATERIAL Y MÉTODOS}

El estudio fue realizado con un enfoque descriptivo y de investigación de campo, generado en la visita al área afectada y a través de entrevistas no estructuradas a los pobladores; así como de la información obtenida de SERFOR en el caso seguido contra la empresa SILMAX E.I.R.L. y la comunidad de Tingopaccha.

Dentro de la metodología se utilizó el método analítico observacional para obtener la información detallada de la realidad que acontecía en la comunidad.

Respecto a la población al 2017, según el testimonio del señor Roel Lionel Villar Rosales, Fiscal de la Comunidad, la población total era de 57 personas entre hombres, mujeres, niños y niñas.

Para la recolección de datos se realizaron entrevistas, trabajo de campo y análisis documental.

\section{RESULTADOS}

En las tablas 1,2, y 3 se plasman las respuestas de 3 comuneros sobre los temas planteados:

Tabla 1

Declaración del Fiscal de la comunidad de Tingopaccha.

\begin{tabular}{l} 
Respuestas: \\
\hline 1. Toda la comunidad sabia del contrato, había gente \\
que no estaba de acuerdo porque querían dinero o \\
sacar provecho. \\
2. En los beneficios, mejoramos nuestro local comunal, \\
el sistema de agua potable y la cancha deportiva. \\
3. En los daños, la denuncia es una mentira, la tierra \\
sigue dando, si quieren los puedo llevar otro día \\
hay papa sembrada les puede hacer ver. \\
\hline Postura: A favor del contrato y desmintió el cambio de \\
uso de suelo.
\end{tabular}


Tabla 2

Declaración de una comunera (nombre reservado).

Respuestas:
1. Toda la gente que aún vive aquí sabia pero no todos
estábamos de acuerdo porque tenemos ganado que
pastear y nos quitan la zona de pasteo al alquilar
2. Mejoraron algunas cosas de la comunidad a
cambio de quitarnos el sustento de cada día.
3. En los daños, es completamente cierto, ya no se
puede pastear por esa zona, suban y miren ustedes
mismos, que no les mientan, ahí no crece nada.
Postura: En contra del contrato, aseguró que hay daños
en la tierra que afectó a los animales.

Tabla 3

Declaración del comunero Luis Flores Molina.

\begin{tabular}{l} 
Respuestas: \\
\hline 1. La gente si sabía, pero los que estaban a favor eran \\
los que se dedican al cultivo y los que estaban en \\
contra son porque son ganaderos. Además, dicen \\
que aparentemente habría una ganancia adicional \\
por parte del presidente de la comunidad. \\
2. es beneficioso para los que cultivan. \\
3. no opino, verifiquen ustedes. \\
Postura: neutral, se abstuvo de brindar mayor \\
información.
\end{tabular}

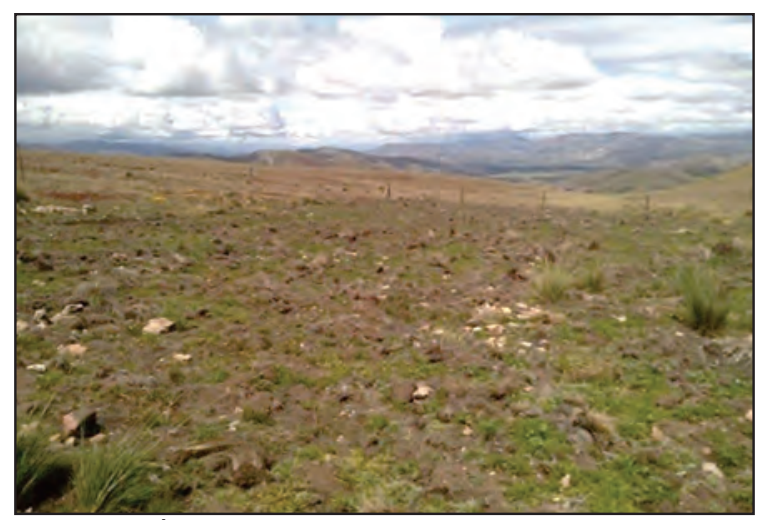

Figura 1. Área afectada por el cambio de uso de suelo

Se verificó que el terreno ubicado en el paraje mencionado líneas arriba, estaba degradado, sin vegetación y cercado con evidentes signos de abandono.

\section{Desarrollo del procedimiento administrativo sancionador}

El 06 de octubre del 2014 el señor Daniel Esteban Borja denunció que la Directiva de la Comunidad Campesina Tingopaccha arrendó ilegalmente 100 ha de terreno comunal a la empresa SILMAX EIRL, quienes destruyeron la vegetación en dicho terreno, lo que dio inicio a un procedimiento administrativo sancionador.
El Informe $N^{\circ}$ 37-2014-SERFOR - ATFFS - SIERRA CENTRAL-SEDE FORESTAL HUANCAYO / VAVM de fecha de 23 de diciembre del 2014 concluyó que efectivamente se constató el cambio de uso de tierra en el paraje Juchupampa en una superficie de 36,45 ha. pertenecientes a la comunidad campesina, incurriendo en la infracción prevista en el inc. e) del artículo $363^{\circ}$ del Reglamento de la Ley Forestal y de Fauna Silvestre. Es así que el SERFOR, mediante Carta $\mathrm{N}^{\circ}$ 011 -2015-SERFOR-ATFFS-Sierra Central de fecha 28 de enero del 2015 inició el procedimiento contra la empresa y la comunidad, por la presunta comisión de la infracción señalada, emitiendo la Resolución Administrativa $\quad N^{\circ}$ 127-2015-SERFOR-ATFFS/ SIERRA CENTRAL de fecha 12 de mayo del 2015; la Administración Técnica Forestal y de Fauna Silvestre (ATFFS) Sierra Central, resolvió imponer una multa de 1.299 UIT (una unidad impositiva con doscientos noventa y nueve centésimos) a los infractores.

Luego de emitida la Resolución Administrativa mencionada, la señora Dina Güere Vega, representante legal de la empresa SILMAX EIRL., interpuso recurso de apelación, el cual fue declarado INFUNDADO por la Dirección de Control de Gestión del Patrimonio Forestal y Fauna Silvestre concluyendo que, en las tierras donde el apelante realizó el cambio de uso, existían formaciones vegetales denominadas pajonales, predominando las especies Stipa Ichu y Festuca Weberbaveri, consideradas Patrimonio Forestal Nacional, conforme al artículo $33^{\circ}$ del Reglamento de la Ley Forestal y de Fauna Silvestre (Ministerio de Agricultura y Riego, 2015); adicionalmente, realizó el cambio de uso en áreas donde no existían estudios de levantamiento de suelos elaborados en base al Reglamento de Clasificación de Tierras según su capacidad de uso mayor, sin contar con la correspondiente autorización otorgada por la ATFFS Sierra Central (arts. $33^{\circ}, 50^{\circ}$, y $287^{\circ}$ del Reglamento de Reglamento de la Ley Forestal y de Fauna Silvestre.

\section{DISCUSIÓN}

El Estado, según la Constitución Política del Perú, tiene la soberanía inminente sobre los recursos naturales y su aprovechamiento, fijando por ley las condiciones de su uso y otorgamiento. Las tierras cubiertas de bosques y otras formaciones vegetales naturales, se consideran parte del Patrimonio Forestal Nacional y solo podrán ser objeto de cambio de otro uso distinto al forestal, previo estudio aprobado por el SERFOR.

En este caso, sumándose al desequilibrio ambiental, está la omisión de la evaluación del expediente técnico de cambio de uso de tierras que afectó también a la vicuña, especie que se encuentra en la categoría de "casi amenazada". (Ministerio de Agricultura y Riego, 2014). 
Por otro lado, expertos del MINAGRI y la Dirección Regional de Agricultura de Junín (DRAJ) determinaron que luego de la siembra de "maca", los pastizales destruidos se comienzan a recuperar a partir de los 10 años, pero con incremento de especies indeseables (no palatables para los camélidos silvestres). Así pues, para recuperar la condición del pastizal inicial es necesario implementar medidas de restauración. En ese sentido, se deben realizar campañas de capacitación a las comunidades alto andinas en el manejo de los recursos forestales conforme a la Ley $N^{\circ} 29763$, para que tengan en cuenta la importancia del manejo del medio ambiente, su conservación y uso sostenible manteniendo el equilibrio ambiental.

\section{Marco Internacional}

a. La Convención de las Naciones Unidas para la Lucha Contra la Desertificación (CNULD) tiene como objetivos: luchar contra la desertificación, mitigar los efectos de la sequía en los países afectados por la sequía grave o desertificación y aplicar estrategias a largo plazo en zonas afectadas concentrando el aumento de la productividad de las tierras, su rehabilitación, conservación y uso sostenible de los recursos de tierras, intentando mejorar las condiciones de vida de sus habitantes. No obstante, el cumplimiento de ésta norma del derecho internacional se limita sólo a los países que hayan suscrito la convención y los protocolos derivados de ésta, y el Perú, como miembro, ha desarrollado herramientas para su cumplimiento, elaborando la Política Nacional del Ambiente y el proyecto de la Estrategia Nacional de Lucha contra la Desertificación y la Sequía 2016 - 2030.

b. El Plan Estratégico Decenal 2008 - 2018 de la Convención de Lucha Contra la Desertificación, adoptado en la Conferencia de las Partes (COP 8) en el que los países miembros adoptaron por unanimidad el Marco y Plan Estratégico Decenal para mejorar la aplicación de la Convención para el período 2008 - 2018, estableciendo objetivos, indicadores y metas a lograr en un período de 10 años, las cuales implican: mejorar las condiciones de vida de las poblaciones y ecosistemas afectados, generar beneficios mundiales mediante la aplicación efectiva de la CNULDS y movilizar recursos para su implementación mediante alianzas eficaces entre agentes nacionales e internacionales.

\section{Marco nacional}

a. La Ley $N^{\circ} 29763$, dispuso la creación del Sistema de Nacional de Gestión Forestal y de Fauna Silvestre (en adelante SINAFOR) como un sistema funcional integrado por los ministerios, organismos e instituciones públicas de los niveles nacional, regional y local que ejercen competencias $y$ funciones en la gestión forestal y de fauna silvestre y por los comités de gestión de bosques reconocidos. Asimismo, creó el SERFOR, ente rector del SINAFOR, encargado de dictar las normas y establecer los procedimientos relacionados a su función fiscalizadora.

De acuerdo a lo investigado, el SERFOR actuó de manera tardía, estableciendo una sanción ínfima que no logró desincentivar la actividad infractora, puesto que se dieron casos dentro de la misma comunidad, que continuó arrendando tierras para el monocultivo de maca.

b. El Reglamento de la Ley Forestal y de Fauna Silvestre, D.S. 020-2015-MINAGRI, modificado a la fecha, señalaba en el artículo 107 numeral 3 literal c) como infracción muy grave realizar el cambio de uso de la tierra sin contar con autorización; y, dentro de su artículo 109 numeral 2 literal c, la sanción comprendía una multa entre 10 hasta 500 UIT.

\section{Marco Local}

El Gobierno Regional Junín realizó la propuesta de implementación de acciones, trabajando de manera conjunta con instituciones privadas y gubernamentales con el fin de combatir la deforestación, la degradación de suelos y la amenaza de la fauna y flora silvestre; en ese sentido, el año 2016 elaboró el Plan de Acción Ambiental al 2021 y de la Política Ambiental Regional.

Es completamente válida la actuación y la preocupación del Gobierno Regional para reducir el porcentaje de tierras degradadas por las actividades mencionadas, con el fin de preservar y conseguir los objetivos planteados dentro del Plan. Sin embargo, en la investigación realizada, no realizó acción alguna para prevenir y recuperar las tierras degradadas de Tingopaccha.

Algunas conclusiones son:

Se concuerda con Carlos Andaluz (2016), que es difícil entender que el Perú carece de un marco legal integrador que regule la protección y el adecuado aprovechamiento de las tierras; las normas y políticas que existen son insuficientes y dispersas; todo ello a pesar de ser un país vinculado fuertemente al agro, tener un territorio altamente sensible a la degradación del suelo, poseer un porcentaje bastante pequeño de tierras aptas para la agricultura y tener tierras para pasturas condicionadas por diversos limitantes naturales.

La presencia del Estado, en todos sus niveles, es mínima en la comunidad de Tingopaccha y es por ello que el problema de desertificación subsiste a 
pesar de que existen herramientas normativas para prevenir, combatir y sancionar. $Y$ ello obedece a la poca eficiencia de los servidores y funcionarios de las entidades competentes.

Con el nuevo reglamento aprobado el 2015, se incrementó la multa por el cambio de uso de tierra sin autorización; sin embargo, la norma no está cumpliendo su finalidad ya que, se siguen arrendando tierras para el monocultivo de maca, lo que es lamentable.

El proyecto de la Estrategia Nacional de Lucha Contra la Desertificación y la Sequía 2016 - 2030, implementada por el MINAM ha sido una respuesta válida por parte del Estado para hacer frente a este problema ambiental. Empero, para que se logre implementar sus objetivos se requiere de especialistas en el tema.

\section{REFERENCIAS BIBLIOGRÁFICAS}

Andaluz, C. (2011). Manual de Derecho Ambiental. Lima: Grijley EIRL.

Google Maps. (2017). Maps. Obtenido de https:// www.google.com.pe/maps/place/1 1\%C2\%B036'4 8.2\%22S+75\%C2\%B033'11.2\%22W/@11.6424, $75.5053113,785 \mathrm{~m} /$ data $=! 3 \mathrm{~m} 1 ! 1 \mathrm{e} 3 ! 4 \mathrm{~m} 5 ! 3 \mathrm{~m} 4$ ! $1 \mathrm{~s}$ $0 \times 0: 0 \times 0 ! 8 m 2 ! 3 d-11.6134 ! 4 d-75.5531 ? \mathrm{hl}=\mathrm{es}$
Ministerio de Agricultura y Riego. (2014). D.S. N ${ }^{\circ}$ 004-2014-MINAGRI. Lima.

Ministerio de Agricultura y Riego. (2015). Decreto Legislativo $N^{\circ} 1220$ - Reglamento de la Ley Forestal y de Fauna Silvestre. Lima.

Ministerio de Agricultura y Riego. (2015). Reglamento de la Ley Forestal y de Fauna Silvestre, D.S. 020-2015-MINAGRI. Lima.

Municipalidad distrital de Acolla. (s.f.). Plan de desarrollo concertado del Dstrito de Acolla 2011-2021. Junin. Jauja: Municipalidad distrital de Acolla. Recuperado el 10 de 2 de 2017, de https://www.scribd.com/document/363677694/ Capitulo-i

Servicio Forestal y de Fauna Silvestre. (2014). Informe N'37- 2014- SERFOR-ATFFS-SIERRA-CENTRALSEDE FORESTAL HUANCAYO/VAVM. Huancayo.

Servicio Nacional de Meteorología e Hidrología del Perú. (2011). Datos climáticos de Diciembre a Abril. Estación Metereológica de Jauja.

Sistema Peruano de la Informacion Jurídica. (1993). Constitución Política del Perú. Obtenido de http:// spij.minjus.gob.pe/libre/main.asp 